\title{
Effects of Mild Traumatic Brain Injury on Stereopsis Detected by a Virtual Reality System: Attempt to Develop a Screening Test
}

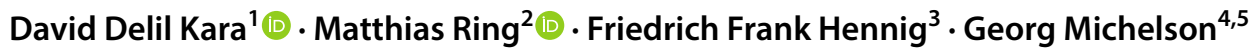

Received: 12 December 2019 / Accepted: 12 June 2020 / Published online: 23 June 2020

(c) The Author(s) 2020

\begin{abstract}
Purpose The study aimed to evaluate stereopsis as a surrogate marker for post-concussion oculomotor function to develop an objective test that can reliably and quickly detect mild traumatic brain injuries (TBI).

Methods The cohort of this prospective clinical study included 30 healthy subjects (mean age $25 \pm 2$ years) and 30 TBI patients ( $43 \pm 22$ years) comprising 11 patients with moderate TBI and 19 patients with mild TBI. The healthy subjects were examined once, whereas the TBI patients were examined immediately after hospitalization, at 1 week, and at 2 months. A virtual reality (VR) program displayed three-dimensional rendering of four rotating soccer balls over VR glasses in different gaze directions. The subjects were instructed to select the ball that appeared to be raised from the screen as quickly as possible via remote control. The response times and fusion abilities in different gaze directions were recorded.

Results The correlation between stereopsis and TBI severity was significant. The response times of the moderate and mild TBI groups were significantly longer than those of the healthy reference group. The response times of the moderate TBI group were significantly longer than those of the mild TBI group. The response times at follow-up examinations were significantly shorter than those immediately after hospitalization. Fusion ability was primarily defective in the gaze direction to the right $\left(90^{\circ}\right)$ and left $\left(270^{\circ}\right.$ and $\left.315^{\circ}\right)$.

Conclusions TBI patients showed impaired stereopsis. Measuring stereopsis in different positions of the visual field using VR can be effective for rapid concussion assessment.
\end{abstract}

Keywords Traumatic brain injury $\cdot$ Concussion $\cdot$ Sports trauma $\cdot$ Clinical assessment $\cdot$ Virtual reality $\cdot$ Stereopsis

Electronic supplementary material The online version of this article (doi:https://doi.org/10.1007/s40846-020-00542-7) contains supplementary material, which is available to authorized users.

David Delil Kara

delil.kara@fau.de

1 Friedrich-Alexander University Erlangen-Nürnberg, Erlangen, Germany

2 Department of Computer Science, Friedrich-Alexander University Erlangen-Nürnberg, Erlangen, Germany

3 Department of Trauma Surgery, Friedrich-Alexander University Erlangen-Nürnberg, Erlangen, Germany

4 Department of Ophthalmology, Friedrich-Alexander University Erlangen-Nürnberg, Erlangen, Germany

5 Talkingeyes + More Gesellschaft mit beschränkter Haftung $(\mathrm{GmbH})$, Erlangen, Germany

\section{Introduction}

Traumatic brain injury (TBI) is a significant health issue particularly among people participating in sports and in the military. TBI is classified as mild, moderate, or severe, with mild TBI or concussion accounting for $75 \%$ of such injuries $[1,2]$. In the United States alone, there are about 3.8 million cases of sports-related mild TBI per year [3-5]. In reality, the incidence is probably much higher; concussions remain underreported because they are often perceived as harmless or athletes choose to continue participation in an event [6]. Frequently, a concussion only becomes apparent when the patient displays symptoms such as headache, photosensitivity, fatigue, irritability, and memory or speech difficulties. However, one study found that many athletes who return to sporting competitions after the resolution of physical symptoms still had anomalies when performing complex oculomotor tasks [7]. 
It is very important to develop an objective test that will detect a mild TBI, both reliably and quickly. Regarding the affected person's health, it is essential to make the correct decision on return-to-play of athletes or return-to-duty of soldiers after a concussion. An athlete who has suffered a concussion has a threefold greater risk of incurring a second concussion in the same season [8]. Furthermore, repeated concussions can lead to long-term sequelae, including depression, neurodegenerative diseases and altered cognition [9-11].

Unfortunately, mild TBI, in particular, poses a major diagnostic challenge due to the lack of objective assessment tools [12]. Traditional medical imaging with computed tomography and magnetic resonance typically reveal no abnormal changes [13-15]. However, concussions lead to oculomotor system disorders, as oculomotor function is regulated by brain areas which are particularly vulnerable to concussions [16-20].

This is the first clinical study to test stereopsis in different gaze directions as a surrogate marker for oculomotor function following concussion. The present study is designed to examine the correlation between stereopsis results in different gaze directions and TBI severity. This study's aim is to develop a rapid method to accurately and objectively detect a mild TBI on the spot with the use of portable virtual reality (VR) glasses.

\section{Materials and Methods}

\subsection{Approval}

The study protocol was approved (serial number: 363_16 B) by the Ethics Committee of the University of Erlangen (Erlangen, Germany) and conducted in accordance with the tenets of the Helsinki Declaration and the "Good Clinical Practice" guidelines. Written informed consent was obtained from all patients before enrollment in this study.

\subsection{Subjects}

The cohort of this prospective clinical study included 30 healthy subjects and 30 patients with mild or moderate TBI. The Glasgow Coma Scale (GCS) was used to assess TBI severity (Electronic Supplementary Material, Table A1). Of the 30 patients, 11 were classified as having moderate TBI (GCS 10-12) and 19 as having mild TBI (GCS 13-15). The mean age of the healthy subjects was $24.73 \pm 2.16$ years (range 19-28 years), whereas that of the patients was $43.07 \pm 22.21$ years (range $17-87$ years). The mean age of patients with mild TBI was $35.84 \pm 17.16$ years (range $17-76$ years), whereas that of patients with moderate TBI was $58.91 \pm 22.70$ years (range $23-87$ years). The sex ratio of the healthy subjects was $18: 12(\mathrm{M}: \mathrm{F})$, of the patients $12: 18$ (M:F). The healthy subjects were examined once, while the TBI patients were examined at three different times: immediately after hospitalization (3-12 h after accident), at 1 week, and at 2 months (Electronic Supplementary Material, Table A2).

We made sure that patients were able to perform the test. In order to eliminate disturbing factors, we have introduced exclusion criteria. The exclusion criteria for study participation included glaucoma, squint surgery, monocular vision, amblyopia, insufficient visual acuity $(<0.7)$, dementia, intoxication, and neurological disorders other than TBI (e.g. attention disturbance, paresis, coordination disorders). Prior to testing, anamnesis was taken to obtain further information about the patient. This also allowed us to assess the mental state of the patient and to evaluate whether his attention and perception was sufficient to perform the test. In order to assess the motor performance and coordination ability, we carried out the muscle function test according to Janda on the upper extremities and the finger-to-nose-test. Both mild and moderate TBI patients showed sufficient coordination ability and a strength level $5 / 5$ according to Janda. Finally, we made sure that the test principle was understood by actively asking the patient to express the test principle in his or her words. Also, the test subjects completed a few trial runs before the actual test.

\subsection{Experimental Setup}

For all tests, a laptop ( $\mathrm{G}$ series; Micro-Star International Co., Ltd., New Taipei City, Taiwan) and VR glasses (VIVE VR System; model no. 99HAHZ0xx-00; HTC Corporation, New Taipei City, Taiwan), with a resolution of $1080 \times 1200$ pixels per eye and a refresh rate of $90 \mathrm{~Hz}$, were used. Two different aspects of vision were tested: central visual acuity and stereopsis.

\subsubsection{Central Visual Acuity as a Basic Examination}

To assess whether visual acuity is sufficient, the central visual acuity of all study participants was determined before measurement of stereopsis using the Landolt ring as a test mark with the Freiburg Visual Acuity Test (FrACT), as described by Michael Bach (Electronic Supplementary Material, Table A3). The Landolt ring is a circle with an opening in one of eight directions (up, down, left, right, and the $45^{\circ}$ positions in between). Visual acuity measurement tests for the smallest sign still recognizable. Visual acuity of each eye was measured independently. The Landolt rings were displayed on the laptop screen, and the study participants were asked to use a remote control from a distance of $2 \mathrm{~m}$ to identify the location of the opening of the rings. 


\subsubsection{Stereopsis as a Parameter for Oculomotor Function}

For this study, a program was created by using the development platform "Unreal Engine" (Electronic Supplementary Material, Fig. A1). The program displayed threedimensional rendering of four rotating soccer balls over VR glasses. The balls were arranged in the form of a rhombus, and each was rotated equally around its own $\mathrm{x}$-axis (Fig. 1). The four balls appeared in random order in one of nine different positions of the visual field (i.e., $0^{\circ}, 45^{\circ}, 90^{\circ}, 135^{\circ}$, $180^{\circ}, 225^{\circ}, 270^{\circ}, 315^{\circ}$ and central) to check stereopsis in different gaze directions. One of the four balls was presented with a different horizontal disparity relative to the other three balls and appeared closer to the subject when the subject was able to perform fusion. The subject was instructed to select this ball as quickly as possible via remote control (four alternative forced choice tests). In the peripheral positions, the balls had an inclination angle of $15^{\circ}$. Three different levels of difficulty were induced by the disparities: 330 , 660, and 990 arcsec. At each position, all three disparities were tested in random order. Each disparity and position was measured five times for a total of 135 measurements by the end of the test.

For this test, fusion (Electronic Supplementary Material, Fig. A2) and two stereoscopic ability parameters were assessed: the response time and correctness of the subject's decision. The timer was automatically started upon presentation of the stimulus and stopped as soon as the subject pressed a button on the remote control. Response times for false decisions were ignored since they do not reflect recognition speeds but the number of errors were documented.

\subsection{Telemedical Approach}

The study participants' oculomotor function was examined via a telemedical approach (Electronic Supplementary Material, Fig. A3). At the examination site, vital parameters were measured, blood samples were collected, and medical history was taken. Visual acuity was measured noninvasively (FrACT) just before oculomotor function assessment by the VR Oculomotor Test.
All patient data were transferred to and stored in MedStage, a cloud-based electronic patient chart, to enable remote viewing by eye specialists for diagnosis. MedStage was developed by Talkingeyes \& More Gesellschaft mit beschränkter Haftung $(\mathrm{GmbH})$ (Erlangen, Germany) and the Tele-Ophthalmology Institute (Erlangen, Germany) and was certified as a medical product class IIa by TÜV Rheinland (Cologne, Germany) in 2018. The collected data and images were then forwarded to the Talkingeyes ${ }^{\circledR}$ Collaboration Network patient record system, where an experienced ophthalmologist telemedically evaluated and diagnosed the fusion visual fields within 2 days of the examination. The medical reports were accessible by the participants within MedStage using personalized login data.

\subsection{Statistical Data Analysis}

All statistical analyses were conducted using IBM SPSS Statistics for Windows, version 25.0 (IBM Corp., Armonk, NY, USA). First, the response times of all gaze directions were analyzed for normal distribution using the Shapiro-Wilk test, which showed that the data were not normally distributed. The descriptive data of response times of all gaze directions are presented as absolute and relative frequencies, as well as 1 st and 3rd quartiles, and median, maximum, and minimum values. Only the correct decisions were included for response time analyses. Then, Spearman's rho and Kendall's tau coefficient were used to identify any bivariate correlation between stereopsis (i.e., response times and error rates) and the GCS score. Differences in the response times, as well as the groups' error rates (i.e., moderate TBI, mild TBI, and healthy reference group), were tested for significance using the Kruskal-Wallis $\mathrm{H}$ test for independent samples. Differences in response times between the different disparities were tested for significance using the Wilcoxon signed-rank test. The significance of the differences in response times, as well as error rates of all patients over the three time points [i.e., immediately after hospitalization (t1), at 1 week (t2), and 2 months (t3)], were assessed using the Wilcoxon signed-rank test for dependent samples. All significance tests were performed for each presented disparity. A probability (p) value of $<0.05$ was considered
Fig. 1 Test set-up and illustration of the stereoscopic stimuli in the central position

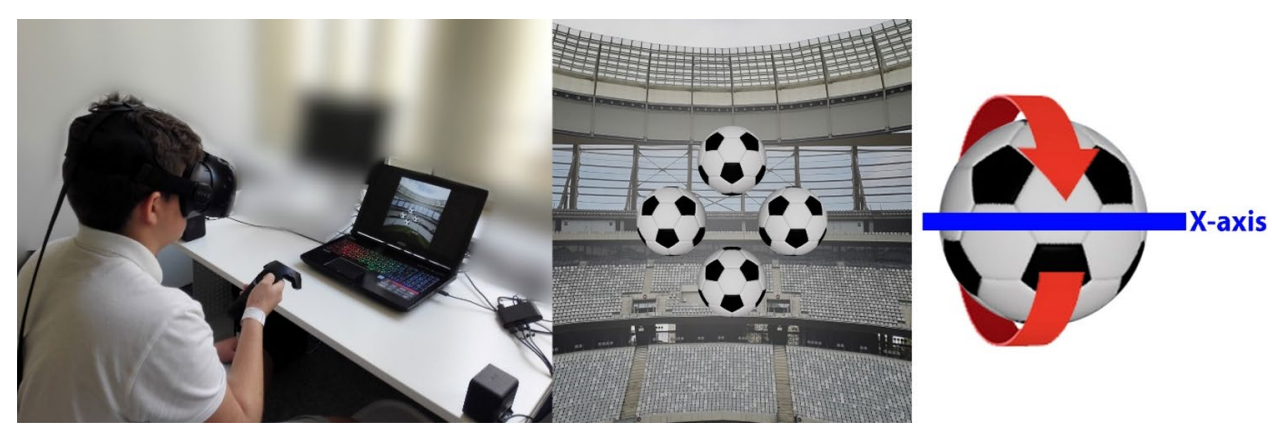


significant, $\mathrm{p}<0.01$ as very significant, and $\mathrm{p}<0.001$ as highly significant. The results were then graphically visualized using Microsoft Excel 2019 (Microsoft Corporation, Redmond, WA, USA).

\section{Results}

\subsection{Correlation}

The correlations between response times or error rates in all gaze directions and the GCS scores were highly significant $(\mathrm{p}<0.001)$.

\subsection{Response Times}

\subsubsection{Initial Examination}

For all three disparities and all gaze directions, the response times of successful fusion of the moderate TBI group, as well as the mild TBI group, were significantly (both $\mathrm{p}<0.001$ ) longer than those of the healthy reference group, while the response times of the patients with moderate TBI were significantly $(p<0.001)$ longer than those of the patients with mild TBI (Fig. 2).In regard to each gaze direction individually (Electronic Supplementary Material, Tables A4-A10), the response times of successful fusion of the moderate TBI group, as well as the mild TBI group, were significantly (both $p<0.001$ ) longer than those of the healthy reference group, while the response times of the patients with moderate TBI were not significantly longer than those of the patients with mild TBI.

\subsubsection{Time Course}

The response times of all patients (moderate and mild TBI) over three time points were compared. For all three disparities and all gaze directions, the response times at t2 were significantly $(\mathrm{p}<0.001)$ shorter than at $\mathrm{t} 1$, while those at $\mathrm{t} 3$ were significantly $(\mathrm{p}<0.001)$ shorter than at $\mathrm{t} 2$ (Fig. 3). In regard to each gaze direction individually, the response times at $\mathrm{t} 3$ were significantly $(\mathrm{p}<0.05)$ shorter than at $\mathrm{t} 1$, while those at $\mathrm{t} 3$ were significantly $(\mathrm{p}<0.05)$ shorter than at $\mathrm{t} 2$, but there was no significant difference between the measurements at $\mathrm{t} 1$ and $\mathrm{t} 2$.

\subsection{Error Rates}

For all three disparities and all gaze directions, the error rates of the moderate TBI group and the mild TBI group were significantly (both $\mathrm{p}<0.001$ ) greater than those of the healthy reference group, while the error rates of the moderate TBI group were not significantly greater than those of the mild TBI group (Fig. 4).

Next, the error rates of all TBI patients over three time points were compared. For all three disparities and all gaze directions, the error rates at 1 week post-accident were significantly $(\mathrm{p}<0.001)$ lower than those immediately after hospitalization. The error rates at 2 months were also significantly lower than those at 1 week (Fig. 5), but the significance levels varied: for the disparity at $330 \mathrm{arcsec}$, the difference was very significant $(\mathrm{p}<0.01)$, whereas for those at 660 and 990 arcsec, the difference was significant $(\mathrm{p}<0.05)$.

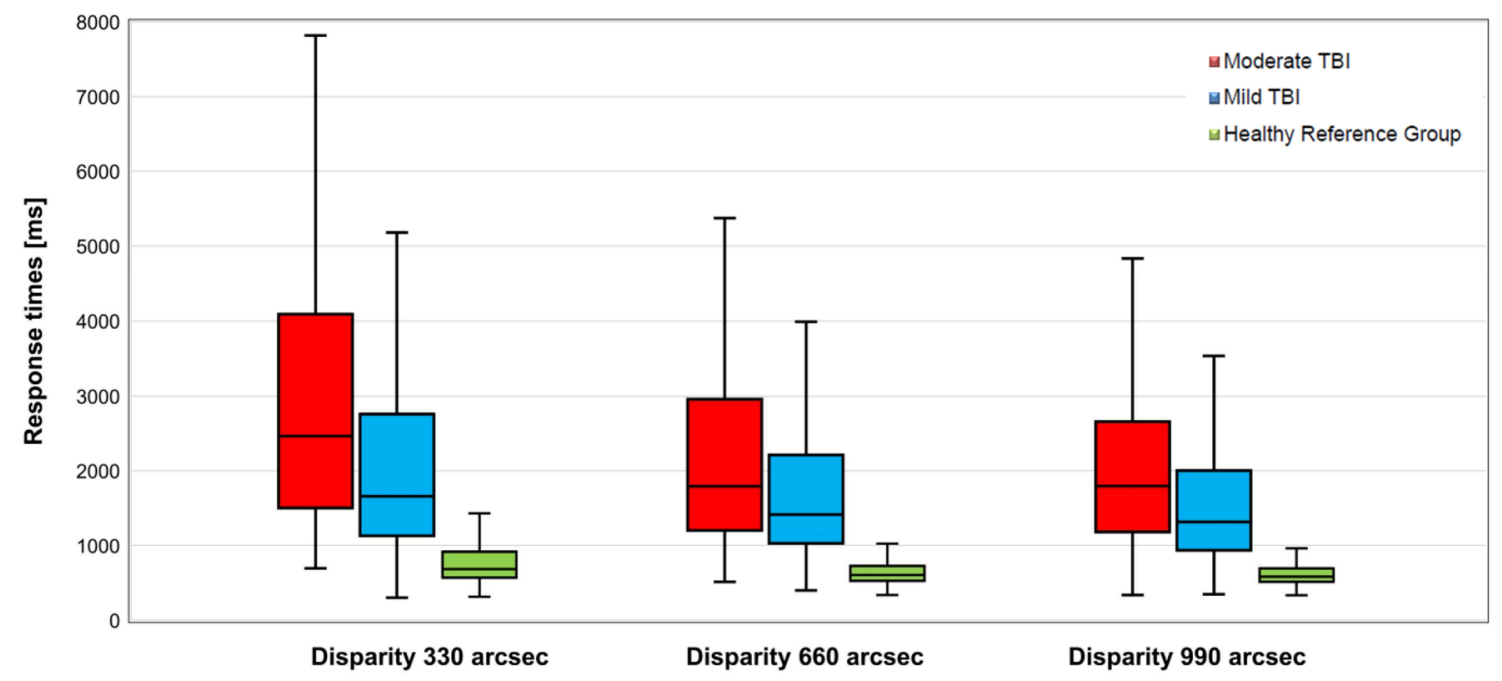

Fig. 2 Response times in all gaze directions immediately after hospitalization vs. the healthy reference group 


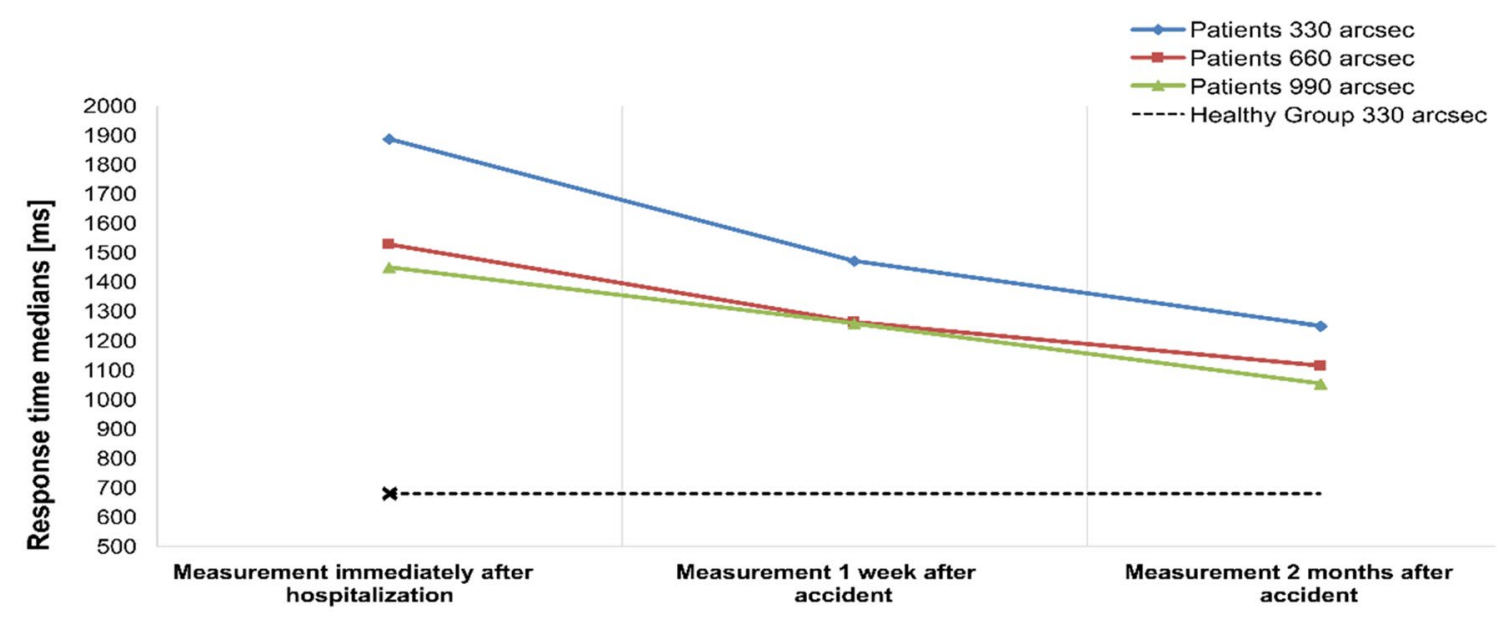

Fig. 3 Monitoring of response time medians

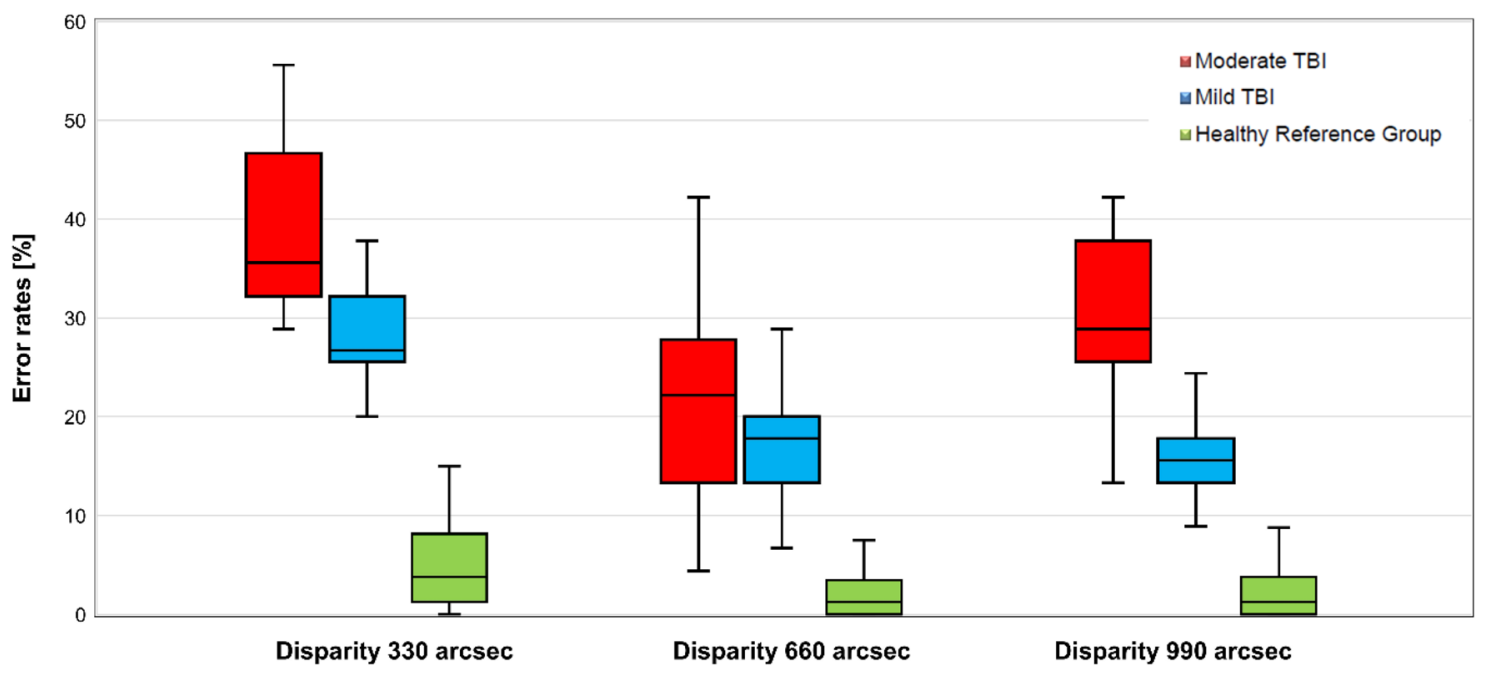

Fig. 4 Error rates of measurements immediately after hospitalization vs. the healthy reference group

\subsection{Response Times of Different Disparities}

In the group of healthy subjects, the response times at 330 arcsec over all gaze directions were significantly $(\mathrm{p}<0.001)$ longer than those at $660 \mathrm{arcsec}$, and those at $660 \mathrm{arcsec}$ were significantly $(\mathrm{p}<0.001)$ longer than those at 990 arcsec. For all TBI patients, the response times immediately after hospitalization were also significantly $(\mathrm{p}<0.001)$ longer at 330 arcsec than at 660 arcsec and significantly $(\mathrm{p}<0.05)$ longer at 660 arcsec than at 990 arcsec (Electronic Supplementary Material, Table A11).

\subsection{Age as Covariate}

Significance tests were conducted in which age as covariate was included. The results did not correlate with the age of patients with TBI. All results remained significant $(\mathrm{p}<0.01)$ taking age into account.

\subsection{Fusion in Different Gaze Directions}

Central fusion was present in all patients. Immediately after hospitalization, 9 (47.4\%) of 19 patients with mild TBI and $9(81.8 \%)$ of 11 patients with moderate TBI had impaired fusion. Mostly, the view to the right (at $90^{\circ}$ ) and the view to the left (at $270^{\circ}$ and $315^{\circ}$ ) showed a lack of fusion (Fig. 6). In total, 8 (26.7\%) of 30 patients had a fusion disorder when looking to the right, and $9(30 \%)$ had a fusion disorder when looking to the left. In all patients, the follow-up measurements at 1 week and 2 months showed partial or complete normalization of the fusion visual field. 


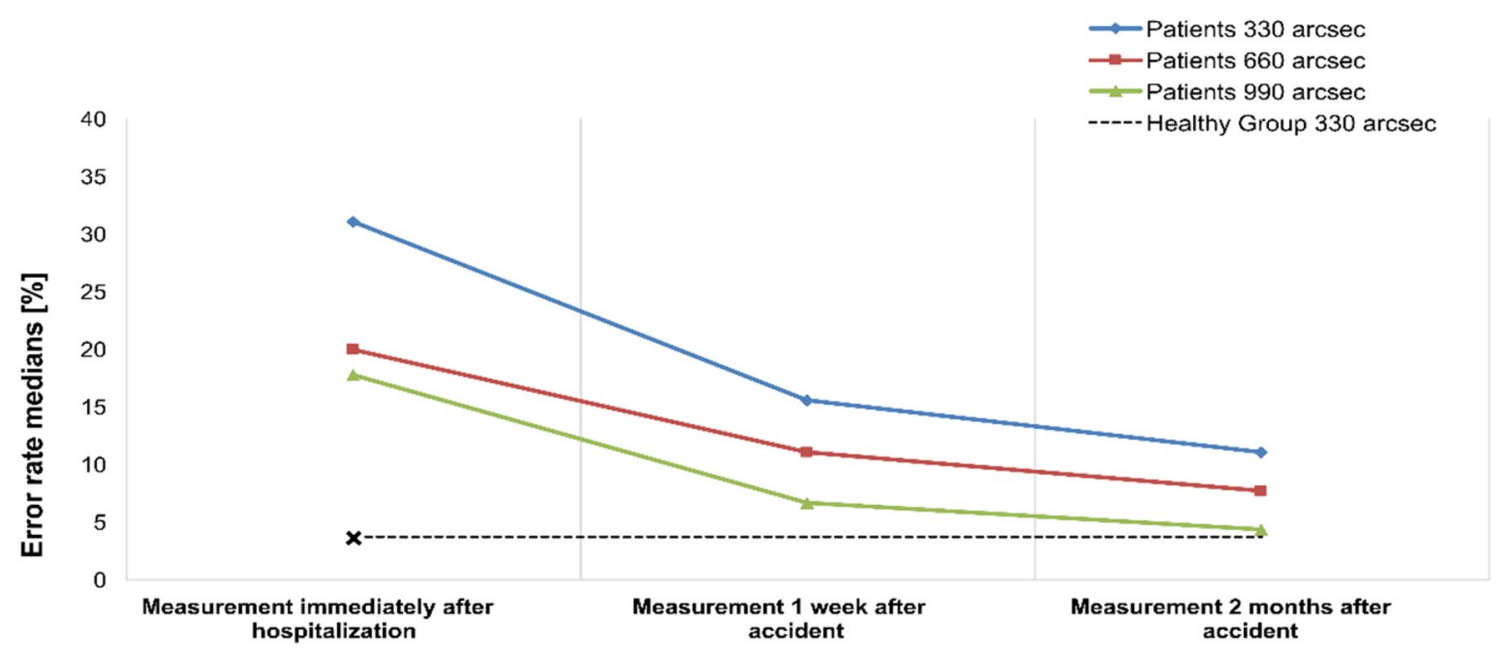

Fig. 5 Monitoring of error rate medians

\section{Fusion visual field deficits in mild TBI patients}

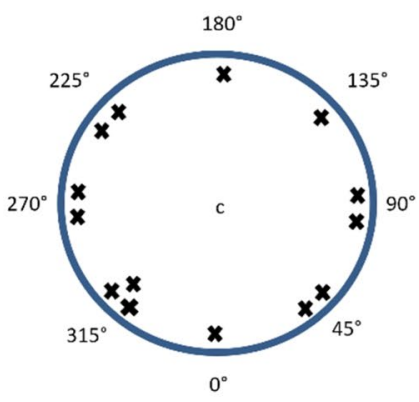

Immediately after hospitalization

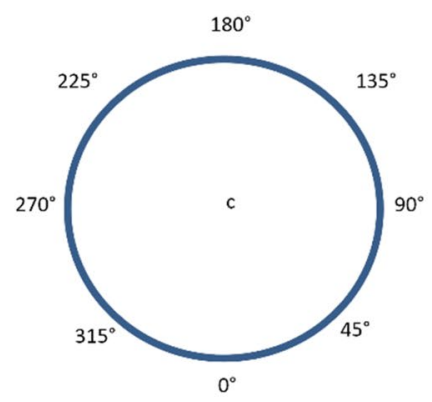

1 week after accident

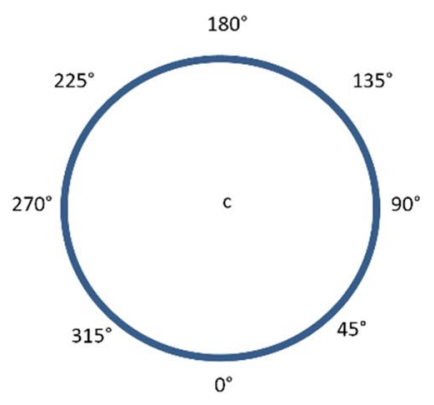

2 months after accident

Fusion visual field deficits in moderate TBI patients

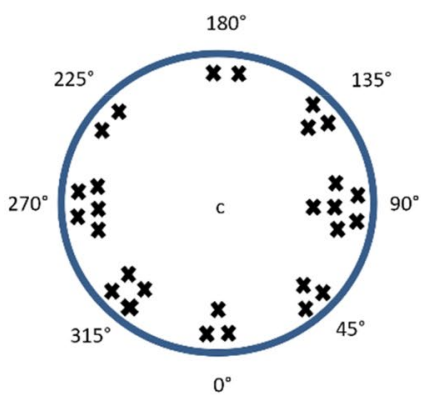

Immediately after hospitalization

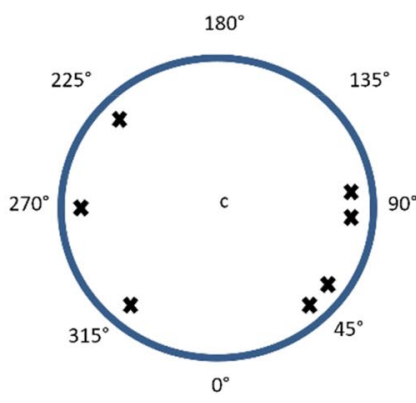

1 week after accident

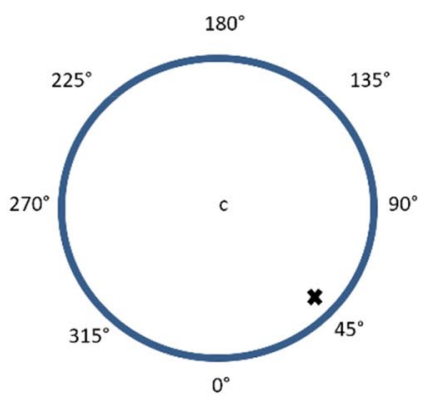

2 months after accident

Fig. 6 The circles represent the fusion visual fields with its nine positions. Each cross represents a fusion deficit per patient and position. $c$ central

\section{Discussion}

To the best of our knowledge, this study is the first to establish stereopsis as a decisive parameter for assessing
TBI severity, and to evaluate both the technical feasibility and clinical applicability of the new VR test. In the present study, a head-mounted display was used to examine the stereoscopic ability by measuring the response time and correctness of the subjects' decisions in eight different 
gaze directions. The proposed method enabled the assessment of the fusion visual field using stimuli of 330, 660, and 990 arcsec with corresponding response times.

The study results confirmed stereopsis as a useful surrogate marker for oculomotor function to assess TBI severity. Our results demonstrated that, compared to healthy subjects, the TBI patients' response times were longer and the error rates of decisions were greater. The prolonged response times and the increased error rates of the concussed patients suggest that a concussion damages the neural pathways that mediate oculomotor function. This finding is consistent with those of earlier studies, which have shown that oculomotor function is regulated by the frontoparietal circuits and subcortical nuclei, which are particularly vulnerable to concussions [16-20]. Concussion leads to a diffuse brain injury, as dysfunction occurs over a wide area of the brain [21]. Since half of the cerebral neuronal connections are involved in the visual process, concussion leads to disorders of the oculomotor system, which could be another explanation for the extended response times and increased error rates [22-24]. The study's strength was the use of the parameter stereopsis as a second-order process, which requires a complex network spanning multiple cortical areas and involving millions of neurons for efficient processing [25, 26].

Moderate TBI caused more defects in fusion than mild TBI. It is very probable that our technique showed TBIinduced alterations of oculomotor function. We were able to confirm that the extent of the impairment of stereopsis was correlated with TBI severity. Patients with moderate TBI had longer response times than patients with mild TBI, which could be explained by the fact that moderate TBI affects neural pathways more strongly than mild TBI. Mostly, the view to the right (at $90^{\circ}$ ) and the view to the left (at $270^{\circ}$ and $315^{\circ}$ ) showed a lack of fusion. This kind of fusion visual field impairment corresponds to an alteration of the abducens nerve.

We observed that smaller disparities were more difficult to discern for both healthy subjects and TBI patients. Hence, the response times were significantly longer and the error rates significantly higher as the disparity decreased. Since the extent of the difficulty was the same in both groups, there is no added value in testing multiple disparities. In future studies, it would be important to select a disparity that is discerned by most of the healthy population, but is still sufficiently low to detect even the mildest TBI. A previous study showed that disparities of 60-90 arcsec were recognized by $75-95 \%$ of healthy subjects [27]. So far, technical possibilities in the field of VR allow the mapping of disparities only at 330 arcsec and greater. Therefore, we recommend a disparity of 330 arcsec.

During the follow-up examinations, the stereopsis impairment gradually improved over a period of 2 months postinjury, reflecting an ongoing recovery process, which is consistent with studies showing that brain recovery takes place after adequate protection [28]. We observed that, even after 2 months, the response times and error rates were clearly greater than the values of the healthy subjects (Figs. 3 and 5). Even if the TBI patients' test results were influenced by a practice effect, they were still worse than those of the healthy reference group. It seemed as if the TBI patients had not fully recovered even after 2 months.

Over the course of the study, we found that the test procedure was well understood by all subjects. Although the VR glasses are a relatively new technological development, and most of the subjects had never worn VR glasses before, all adapted well to the device regardless of age. The applied system using VR glasses was well tolerated and did not lead to any side effects, such as nausea, dizziness or headache, which may have resulted in discontinuation of testing. A major advantage of this method is its portability, which allowed us to perform the test even at the patient's bedside. This method is, therefore, very well suited for use along the side line during a sporting event and would be available for immediate use in case of concussion.

In emergency situations there are often no neurologists or ophthalmologists on site. The telemedical connection of the virtual reality system enables a doctor to validate the findings remotely. In addition, the patient can see for himself whether there is no, mild or moderate oculomotor dysfunction. This enables the patient to better understand the severity of his concussion and thus increases the patient's understanding of the disease and his compliance to therapy. Furthermore, it is possible to monitor a TBI objectively, so the recovery of the concussed brain can be supervised and, in the absence of regeneration, an adjustment of the therapy can be made.

There are four study limitations. First, despite the large number of subjects, the sample size was still too small to determine significant differences in error rates between the mild and moderate TBI group. Second, the patients were not tested immediately after the accident, but rather immediately after hospitalization, so the test results may not accurately reflect the exact values of an acutely concussed brain. Third, the subjects' education levels were not taken into consideration, which may have influenced the test results. Lastly, it should be mentioned that the healthy reference group consisted of young students, while the patient group included individuals of various ages. This is especially important because spatial vision develops in childhood and begins to decrease from the age of 65 years [29, 30]. Since we also had patients older than 65 , some test values may have been affected by the physiological decrease of stereopsis in old age. Hence, it is advisable to introduce an age criterion in future studies.

Further large-scale trials are required to confirm our results and define reference ranges in order to create an 
exclusionary test for traumatic brain injuries and to determine the sensitivity of this exclusionary test. This screening test can also be coupled with pupillometry to optimize the detection of TBI [31-33].

\section{Conclusions}

The proposed VR System could detect initial oculomotor impairment and impairment over time. Second, the VR System was found to be appropriate for telemedical evaluation. Timely concussion detection is very important since adequate treatment can protect the patient from serious health consequences. Since oculomotor function impairment is subtle, and spatial vision is essential in sports and in the military, stereopsis assessment is suitable for return-to-play/ return-to-duty decisions. The study's findings confirmed that measuring stereopsis using VR glasses is an effective neuroophthalmological tool for concussion assessment.

Acknowledgements Open Access funding provided by Projekt DEAL. We thank all patients, students, and physicians of the Trauma Surgery Department for their participation in this study. The author, David Delil Kara, is a former medical student at Friedrich-Alexander-Universität Erlangen-Nürnberg (FAU), and the present work is submitted as a partial fulfillment of the requirements for a Doctor of Medicine degree.

Funding The authors received no specific funding for this work.

\section{Compliance with Ethical Standards}

Conflict of interest Georg Michelson is the owner of Talkingeyes \& More GmbH. David Delil Kara, Matthias Ring, and Friedrich Frank Hennig declare that they have no conflict of interest.

Open Access This article is licensed under a Creative Commons Attribution 4.0 International License, which permits use, sharing, adaptation, distribution and reproduction in any medium or format, as long as you give appropriate credit to the original author(s) and the source, provide a link to the Creative Commons licence, and indicate if changes were made. The images or other third party material in this article are included in the article's Creative Commons licence, unless indicated otherwise in a credit line to the material. If material is not included in the article's Creative Commons licence and your intended use is not permitted by statutory regulation or exceeds the permitted use, you will need to obtain permission directly from the copyright holder. To view a copy of this licence, visit http://creativecommons.org/licenses/by/4.0/.

\section{References}

1. Gerberding, J. L., \& Binder, S. (2018). The Report to Congress on Mild Traumatic Brain Injury in the United States: Steps to Prevent a Serious Public Health Problem. Retrieved November 1, 2018 from https://www.cdc.gov/traumaticbraininjury/pdf/mtbir eport-a.pdf
2. Centers for Disease Control and Prevention. (2018). Injury prevention \& control: traumatic brain injury \& concussion. Retrieved November 1, 2018 from http://www.cdc.gov/traumaticbraininjury /data/rates.html

3. Meehan, W. P., III., \& Micheli, L. J. (2011). Concussion results in deficits in neurocognitive functioning. Preface Clinical Sports Medicine, 30, xvii. https://doi.org/10.1016/j.csm.2010.09.008

4. Langlois, J. A., Rutland-Brown, W., \& Wald, M. M. (2006). The epidemiology and impact of traumatic brain injury: A brief overview. Journal of Head Trauma Rehabilitation, 1, 375-378.

5. Langlois, J.A.R.-B.W., \& Thomas, K. E. (2004). Traumatic brain injury in the United States: Emergency department visits, hospitalizations, and deaths. Atlanta: Centers for Disease Control and Prevention, National Center for Injury Prevention and Control.

6. Torres, D. M., Galetta, K. M., Phillips, H. W., et al. (2013). Sportsrelated concussion: Anonymous survey of a collegiate cohort. Neurology Clinical Practice, 3, 279-87. https://doi.org/10.1212/ CPJ.0b013e3182a1ba22

7. Baker, C. S., \& Cinelli, M. E. (2014). Visuomotor deficits during locomotion in previously concussed athletes 30 or more days following return to play. Physiological Reports, 2, e12252. https:// doi.org/10.14814/phy2.12252

8. Guskiewicz, K. M., Weaver, N. L., Padua, D. A., et al. (2000). Epidemiology of concussion in collegiate and high school football players. American Journal of Sports Medicine, 28, 643-650. https ://doi.org/10.1177/03635465000280050401

9. Halstead, M. E., \& Walter, K. D. (2010). Council on sports medicine and fitness. American Academy of Pediatrics. Clinical report- sport-related concussion in children and adolescents. Pediatrics, 126, 597-615. https://doi.org/10.1542/peds.2010-2005

10. Sim, A., Terryberry-Spohr, L., \& Wilson, K. R. (2008). Prolonged recovery of memory functioning after mild traumatic brain injury in adolescent athletes. Journal of Neurosurgery, 108, 511-516. https://doi.org/10.3171/JNS/2008/108/3/0511

11. Gavett, B. E., Cantu, R. C., \& Shenton, M. (2011). Clinical appraisal of chronic traumatic encephalopathy: Current perspectives and future directions. Current Opinion in Neurology, 24, 525-531. https://doi.org/10.1097/WCO.0b013e32834cd477

12. Schmid, K. E., \& Tortella, F. C. (2012). The diagnosis of traumatic brain injury on the battlefield. Frontiers in Neurology, 3, 90. https://doi.org/10.3389/fneur.2012.00090

13. Pulsipher, D. T., Campbell, R. A., Thoma, R., et al. (2011). A critical review of neuroimaging applications in sports concussion. Current Sports Medicine Reports, 10, 14-20. https://doi. org/10.1249/JSR.0b013e31820711b8

14. Sasaki, T., Pasternak, O., \& Mayinger, M. (2014). Hockey concussion education project, Part 3 . White matter microstructure in ice hockey players with a history of concussion: a diffusion tensor imaging study. Journal of Neurosurgery, 120, 882-890. https:// doi.org/10.3171/2013.12.JNS132092

15. Grossman, E. J., Jensen, J. H., \& Babb, J. S. (2013). Cognitive impairment in mild traumatic brain injury: a longitudinal diffusional kurtosis and perfusion imaging study. American Journal of Neuroradiology, 34, 951-957. https://doi.org/10.3174/ajnr.A3358

16. White, O. B., \& Fielding, J. (2012). Cognition and eye movements: Assessment of cerebral dysfunction. Journal of Neuro-Ophthalmology, 32, 266-273. https://doi.org/10.1097/WNO.0b013e3182 688230

17. Nevin, N. C. (1967). Neuropathological changes in the white matter following head injury. Journal of Neuropathology \& Experimental Neurology, 26, 77-84.

18. Lipton, M. L., Kim, N., \& Park, Y. K. (2012). Robust detection of traumatic axonal injury in individual mild traumatic brain injury patients: intersubject variation, change over time and bidirectional changes in anisotropy. Brain Imaging and Behavior, 6, 329-342. https://doi.org/10.1007/s11682-012-9175-2 
19. Maruta, J., Lee, S. W., Jacobs, E. F., et al. (2010). A unified science of concussion. Annals of the New York Academy of Sciences, 1208, 58-66. https://doi.org/10.1111/j.1749-6632.2010.05695.x

20. Rucker, J. C., Buettner-Ennever, J. A., Straumann, D., et al. (2019). Case studies in neuroscience: Instability of the visual near triad in traumatic brain injury-evidence for a putative convergence integrator. Journal of Neurophysiology, 122(3), 1254-1263. https ://doi.org/10.1152/jn.00861.2018

21. Hardman, J. M., \& Manoukian, A. (2002). Pathology of head trauma. Neuroimaging Clinics of North America, 12, 175-187.

22. Ciuffreda, K. J., Kapoor, N., Rutner, D., et al. (2007). Occurrence of oculomotor dysfunctions in acquired brain injury: a retrospective analysis. Optometry, 78, 155-161. https://doi.org/10.1016/j. optm.2006.11.011

23. Hunt, A. W., Mah, K., Reed, N., et al. (2015). Oculomotor- based vision assessment in mild traumatic brain injury: A systematic review. Journal of Head Trauma Rehabilitation, 31, 252-261. https://doi.org/10.1097/HTR.0000000000000174

24. Felleman, D. J., \& Van Essen, D. C. (1991). Distributed hierarchical processing in the primate cerebral cortex. Cerebral Cortex, 1 , $1-47$.

25. Cumming, B. G., \& DeAngelis, G. C. (2001). The Physiology of Stereopsis. Annual Review of Neuroscience, 24, 203-238.

26. Welchman, A. E. (2016). The human brain in depth: How we see in 3D. Annual Review of Vision Science, 2, 345-376. https://doi. org/10.1146/annurev-vision-111815-114605
27. Paulus, J., Tong, J., \& Michelson, G. (2014). Extended stereopsis evaluation of professional and amateur soccer players and subjects without soccer background. Frontiers in Psychology, 5, 1186. https://doi.org/10.3389/fpsyg.2014.01186

28. Meier, T. B., Bellgowan, P. S., \& Singh, R. (2015). Recovery of cerebral blood flow following sports-related concussion. JAMA Neurology, 72, 530-538. https://doi.org/10.1001/jamaneurol .2014 .4778

29. Plourde, M., Corbeil, M. E., \& Faubert, J. (2017). Effect of age and stereopsis on a multiple-object tracking task. PLOS ONE, 12, e0188373. https://doi.org/10.1371/journal.pone.0188373

30. Faubert, J. (2002). Visual perception and aging. Canadian Journal of Experimental Psychology, 56, 164-176.

31. Taylor, W. R., Chen, J. W., Meltzer, H., et al. (2003). Quantitative pupillometry, a new technology: Normative data and preliminary observations in patients with acute head injury. Technical note. Journal of Neurosurgery, 98, 205-213.

32. Mohan, K., Kecova, H., Hernandez-Merino, E., et al. (2013). Retinal ganglion cell damage in an experimental rodent model of blast-mediated traumatic brain injury. Investigative Ophthalmology \& Visual Science, 98, 205-213. https://doi.org/10.3171/ jns.2003.98.1.0205

33. Capo-Aponte, J., Urosevich, T. G., Walsh, D. V., et al. (2013). Pupillary light reflex as an objective biomarker for early identification of blast-induced TBI. The Spine Journal. https://doi. org/10.4172/2165-7939.S4-004 